\title{
Vigilancia de los serotipos y susceptibilidad antimicrobiana de Haemophilus influenzae en Colombia, 1994-2002
}

\author{
María Victoria Ovalle ${ }^{1}$, Clara I. Agudelo ${ }^{1}$, Nélida Muñoz ${ }^{1}$, Elizabeth Castañeda ${ }^{1}$, Carmen R. \\ Gallego ${ }^{2}$, Sandra Núñez ${ }^{2}$, Edilma Jaramillo ${ }^{3}$, Vianey Emilce Portilla ${ }^{4}$, Mercedes Cano ${ }^{5}$, \\ Martha Gartner ${ }^{6}$, María Helena Alvarez ${ }^{7}$, Gladys Mora ${ }^{8}$, Patricia Rincón ${ }^{9}$, Martha Uzeta ${ }^{10}$ \\ ${ }^{1}$ Grupo de Microbiología, Instituto Nacional de Salud, Bogotá, D.C., Colombia. \\ ${ }^{2}$ Laboratorio de Salud Pública, Bogotá, D,C, Colombia. \\ ${ }^{3}$ Laboratorio de Salud Pública, Medellín, Antioquia, Colombia. \\ ${ }^{4}$ Laboratorio de Salud Pública, Bucaramanga, Santander, Colombia. \\ ${ }^{5}$ Laboratorio de Salud Pública, Pereira, Risaralda, Colombia. \\ ${ }^{6}$ Laboratorio de Salud Pública, Cali, Valle, Colombia. \\ ${ }^{7}$ Laboratorio de Salud Pública, Manizales, Caldas, Colombia. \\ ${ }^{8}$ Laboratorio de Salud Pública, Cúcuta, Norte de Santander, Colombia. \\ ${ }^{9}$ Hospital de La Misericordia, Bogotá, D.C., Colombia. \\ ${ }^{10}$ Clínica del Niño del ISS, Bogotá, D.C., Colombia
}

La enfermedad invasora causada por Haemophilus influenzae, serotipo b, ha sido una de las mayores causas de morbilidad y mortalidad en la población infantil; afortunadamente, en algunos países con amplia cobertura de la vacuna conjugada esta situación ha cambiado. En 1994 se inició en el Grupo de Microbiología un programa de vigilancia de la susceptibilidad antimicrobiana y de los serotipos de aislamientos invasores de $H$. influenzae, remitidos por los hospitales y Laboratorios de Salud Pública del país como componente de los programas de vigilancia en red de infección respiratoria aguda y meningitis bacteriana aguda. El objetivo de este trabajo fue determinar la evolución de los serotipos y los patrones de susceptibilidad antimicrobiana de los aislamientos invasores de $H$. influenzae obtenidos de 1994 al 2002 y realizar un nuevo análisis sobre el impacto de la vacuna conjugada de $\mathrm{H}$. influenzae, serotipo b, en Colombia. De 1994 a 2002 se han estudiado 683 aislamientos; 379 (55,5\%) de pacientes del género masculino; 370 (54,2\%) de menores de 1 año; 227 (33,2\%) de 1 a 5 años; 19 (2,8\%) de 6 a 14 años; 38 (5,6\%) de mayores de 14 años, y de $29(4,2 \%)$ no se tenía el dato de la edad; $493(72,2 \%)$ fueron recuperados de pacientes con meningitis, $181(26,5 \%)$ de neumonía y 9 $(0,9 \%)$ de otras enfermedades. El $85,1 \%$ de los aislamientos fueron $H$. influenzae, serotipo b, $12,9 \%$ no capsulares y $2,0 \%$ de otros serotipos ( $10 \mathrm{a}, 1 \mathrm{~d}, 1$ e y $2 \mathrm{f}$ ). Del total de aislamientos, $12 \%$ fueron productores de beta-lactamasa; $13,9 \%$, resistentes a ampicilina; $12,7 \%$, a trimetoprim sulfametoxazol (SXT); 5,4\%, a cloranfenicol, y $1 \%$ a cefuroxima; todos fueron sensibles a ceftriaxona. Durante este período se observó un incremento en la resistencia de los aislamientos a SXT ( $5 \%$ al $13 \%)$, pero la diferencia no fue estadísticamente significativa $(p=0,1)$. Con la vigilancia se pudo determinar una disminución significativa de los casos de meningitis en los menores de 1 año y en el grupo de 1 a 5 años; antes de la introducción de la vacuna de Hib, se recibía un promedio anual de 43 y 23 aislamientos de cada uno de estos grupos y en el 2002 se recibieron 10 y 6 , respectivamente $(p<0,001)$. La vigilancia de los aislamientos invasores de $H$. influenzae ha permitido evaluar el impacto de la vacuna de $H$. influenzae, serotipo b, determinar el incremento de aislamientos no capsulares causantes de enfermedad invasora y los cambios en los patrones de resistencia.

Palabras clave: Haemophilus influenzae, vigilancia por laboratorio, serotipos, susceptibilidad antimicrobiana, población infantil, vacunación.

Surveillance of Haemophilus influenzae serotypes and antimicrobial resistance in Colombia, 1994-2002

Invasive disease caused by Haemophilus influenzae serotype b results in high rates of morbidity and mortality among children. In 1994, the Microbiology Group at the Instituto Nacional de 
Salud (Colombia) initiated a program to detect antimicrobial resistance in $H$. influenzae. Invasive isolates were collected by hospitals and public health laboratories as part of surveillance programs for acute respiratory infections and acute bacterial meningitis. To determine the evolution of serotypes and antimicrobial resistance patterns, invasive $H$. influenzae isolates collected from 1994 to 2002 were compared, and the impact of Hib conjugated vaccine in Colombia was reassessed. The analysis included 683 isolates, 379 (55.5\%) were recovered from male patients, $370(54.2 \%)$ from children under one year, $227(33,2 \%)$ from children aged 1 to $5,19(2.8 \%)$ from children aged 6 to 14, and 38 (5.6\%) from children over 14 years; 29 $(4.2 \%)$ with no age data. Clinical classification recorded $493(72.2 \%)$ of the samples were from patients with meningitis, 181 (26.5\%) with pneumonia, and $9(0.9 \%)$ with other diseases. Eighty five percent of isolates corresponded to $H$. influenzae serotype $b, 12.9 \%$ were non capsular, and $2.0 \%$ corresponded to other serotypes ( $10 \mathrm{a}, 1 \mathrm{~d}, 1 \mathrm{e}$ and $2 \mathrm{f}$ ). Of the total number of isolates, $12.0 \%$ produced beta lactamase, $13.9 \%$ were resistant to ampicillin, $12.7 \%$ to trimethoprim sulfamethoxazole (SXT), $5.4 \%$ to chloramphenicol, $1 \%$ to cefuroxime. All isolates were susceptible to ceftriaxone. During the 10-year period, resistance to SXT increased from $5 \%$ to $13 \%$. A significant decrease in meningitis cases was detected among children under one-year old and in the 1 to 5 age group. Before introducing the vaccine, an annual average of 43 and 23 isolates for each of these groups were received. During 2002, 10 and 6 isolates, respectively, were received for each group. Surveillance of invasive $H$. influenzae isolates has allowed the evaluation of Hib vaccine impact, as well as the detection of an increase of non-capsular isolates, and changes in resistance patterns.

Key words: Haemophilus influenzae, laboratory surveillance, serotypes, antimicrobial susceptibility, children, vaccination.

Haemophilus influenzae es un cocobacilo Gram negativo que forma parte de la flora normal del tracto respiratorio humano que, sin embargo, constituye uno de los principales agentes etiológicos de una variedad de enfermedades en la población pediátrica, tales como meningitis, neumonía, otitis media y epiglotitis $(1,2)$. Estas infecciones son causa de alta morbilidad y mortalidad en esa población, especialmente en países en vías de desarrollo, donde representan una carga importante en los gastos de salud, especialmente en los países en donde no se ha incluido la vacuna conjugada en el Programa Ampliado de Inmunizaciones, PAI (2-4).

Existen seis tipos capsulares de $H$. influenzae denominados de la a a la $f$, de los cuales el serotipo $b$ es el principal causante de meningitis y neumonía en niños menores de cinco años. Se ha estimado que $H$. influenzae tipo b ocasionaba en el mundo antes de la era de la vacunación

\section{Correspondencia:}

Clara Inés Agudelo, Avenida calle 26 No. 51-60, Bogotá, D.C. Teléfono: (571) 22077 00, extensión 445

cagudelov@ins.gov.co

Recibido: 11/03/03; aceptado: 27/05/03 cerca de tres millones de casos de enfermedad por año, con una mortalidad de $15 \%$ a $23 \%(3,5)$. Los otros serotipos han estado relacionados con infecciones del tracto respiratorio bajo y se pueden aislar en casos de sinusitis, otitis y conjuntivitis, pero casi nunca han causado infecciones con compromiso sistémico (1-5).

Adicionalmente, la resistencia de los aislamientos de $H$. influenzae, serotipo $b$, a diversos antimicrobianos como cloranfenicol, ampicilina, trimetoprim-sulfametoxazol (SXT) y cefalosporinas de tercera generación, se ha convertido en un problema que aumenta el costo de la terapia y la posibilidad del fracaso del tratamiento $(6,7)$.

Dada la importancia de este patógeno para la salud pública, el Grupo de Microbiología del Instituto Nacional de Salud inició en 1994 una vigilancia en red con los Laboratorios de Salud Pública (LSP) y los hospitales del país para determinar los serotipos circulantes y los patrones de susceptibilidad antimicrobiana de los aislamientos invasores de $H$. influenzae, como parte de los programas de vigilancia en red de la infección respiratoria aguda (IRA) y la meningitis bacteriana aguda (MBA) $(8,9)$. 
La introducción de la vacuna conjugada para $H$. influenzae, serotipo b, desde 1990 en algunos países de Latinoamérica como Uruguay y Chile, ha demostrado ser una buena intervención; la enfermedad causada por $H$. influenzae, serotipo b, ha disminuido entre $90 \%$ y $98 \%(10,11)$, al igual que lo hizo en los países desarrollados (12). En Colombia, la vacuna se incluyó en el PAI en mayo de 1998 en los niños menores de 1 año; en junio de 2000 se había alcanzado una cobertura de $50 \%$ y una disminución de $50 \%$ de los casos de meningitis por $H$. influenzae, serotipo b, en este grupo de edad (13). Posteriormente, se observó que con una cobertura de vacunación de entre $30 \%$ y $45 \%$ se podría prevenir un buen número de casos de neumonías graves, lo que demuestra que la vacuna tiene una eficacia de $56 \%$ (14).

El objetivo de este estudio fue determinar la evolución de los serotipos y los patrones de susceptibilidad antimicrobiana de $H$. influenzae entre 1994 y 2002, así como realizar un nuevo análisis sobre el impacto de la vacuna conjugada H. influenzae, serotipo b, en Colombia.

\section{Materiales y métodos}

\section{Sistema de vigilancia}

En 1994 se estableció el sistema de vigilancia por el laboratorio, en cuyo marco los laboratorios de los hospitales del país deben remitir, con los datos mínimos demográficos y de procedencia, todos los aislamientos invasores de $\mathrm{H}$. influenzae a los LSP para allí ser confirmados y posteriormente enviados al Grupo de Microbiología del Instituto Nacional de Salud.

Se analizó la distribución de los aislamientos invasores de H. influenzae por año, departamento, serotipos y por patrón de susceptibilidad antimicrobiana.

Aislamientos. Todos los aislamientos se confirmaron por coloración de Gram, morfología típica de las colonias en agar chocolate, prueba de requerimiento de factores utilizando tiras impregnadas de factores $\mathrm{V}$ y $X$ (Difco) y prueba de porfirinas (15). Los aislamientos fueron serotipificados con el método de aglutinación en lámina con antisueros polivalentes y monovalentes de la a a la $f$ (Difco) $(15,16)$.
Susceptibilidad antimicrobiana. Se determinó la producción de la $\beta$ lactamasa por el método cromogénico, utilizando discos de cefinasa (BBL). También se determinó la susceptibilidad antimicrobiana por el método de difusión de disco (Kirby-Bauer), en agar Haemophilus test medium (HTM) (Oxoid CM337) suplementado con hematina y ácido delta-amino-levulínico (NAD) (Oxoid SR158E). Se utilizaron discos de ampicilina $(10 \mu \mathrm{g})$, cloranfenicol $(30 \mu \mathrm{g})$, SXT $(1,25 / 23,75 \mu \mathrm{g})$, cefuroxima $(30 \mu \mathrm{g})$ y ceftriaxona $(30 \mu \mathrm{g})$ (Difco). Para el procedimiento y la interpretación se siguieron las recomendaciones del Comité Nacional de Estándares de Laboratorio de Estados Unidos (NCCLS) (17).

En los aislamientos resistentes por el método de Kirby-Bauer se determinó la concentración inhibitoria mínima (CIM), utilizando el método de microdilución en caldo Mueller Hinton II ajustado con cationes (BBL) y suplementado con extracto de levadura, NAD y hematina, de acuerdo con las recomendaciones de la NCCLS (18). Para la interpretación de la CIM se utilizaron los parámetros de la NCCLS (18).

Control de calidad externo. El control de calidad externo es realizado por la Unidad de Referencia de Haemophilus del Laboratorio de Salud Pública (PHLS) de Oxford, Reino Unido. Para las pruebas de susceptibilidad antimicrobiana se utilizó como cepa control H. influenzae ATCC 49247.

Evaluación del impacto de la vacuna. Para el análisis estadístico se calculó el número de casos por año de meningitis por $\mathrm{H}$. influenzae, serotipo b, en menores de 1 año y en los niños de 1 a 5 años antes de la vacunación y luego se calculó el número de casos esperados después de la vacunación con base en el promedio anual en la misma población. La significancia estadística de la diferencia de los dos valores se calculó por medio de la aproximación de la distribución de Poisson a la distribución normal.

Análisis estadístico. Se realizó utilizando el programa Epi-Info 6.1

\section{Resultados}

Aislamientos. De enero de 1994 a diciembre de 2002 se confirmaron 683 aislamientos invasores 
procedentes de 16 departamentos y del Distrito Capital. En el cuadro 1 se observa la distribución por departamento y por año.

Por enfermedad invasora, 493 (72,2\%) aislamientos eran de pacientes con meningitis, $181(26,5 \%)$ de neumonía y $9(1,3 \%)$ de otras patologías (8 sepsis y 1 artritis); 490 (71,7\%) fueron recuperados de LCR, $181(26,5 \%)$ de hemocultivos y $12(1,8 \%)$ de líquido pleural.

Edad. La distribución de los aislamientos de $H$. influenzae por grupos de edad de los pacientes se consigna en la figura $1 ; 370(54,2 \%)$ fueron menores de 1 año y, de ellos, $225(60,8 \%)$ eran niños de 0 a 6 meses. De los 683 aislamientos, $379(55,5 \%)$ eran de pacientes del género masculino y $304(44,5 \%)$ del femenino. Esta distribución fue similar en todos los grupos de edad.

Serotipos. El predominante fue el b, en 581 $(85,1 \%)$ aislamientos, seguido del serotipo a en $10(1,5 \%)$ y $4(0,5 \%)$ de otros serotipos ( $1 \mathrm{~d}, 1$ e y $2 \mathrm{f}) ; 88(12,9 \%)$ aislamientos fueron no capsulares (NC). La distribución de los aislamientos de serotipos b y NC por diagnóstico y por grupos de edad se consigna en el cuadro 2.

En el grupo de edad de menores de 15 años se encontró que el serotipo b estaba más asociado con meningitis, $445 / 542(82,1 \%)$ y el NC con neumonía, 39/63 (61,9\%). A diferencia del grupo

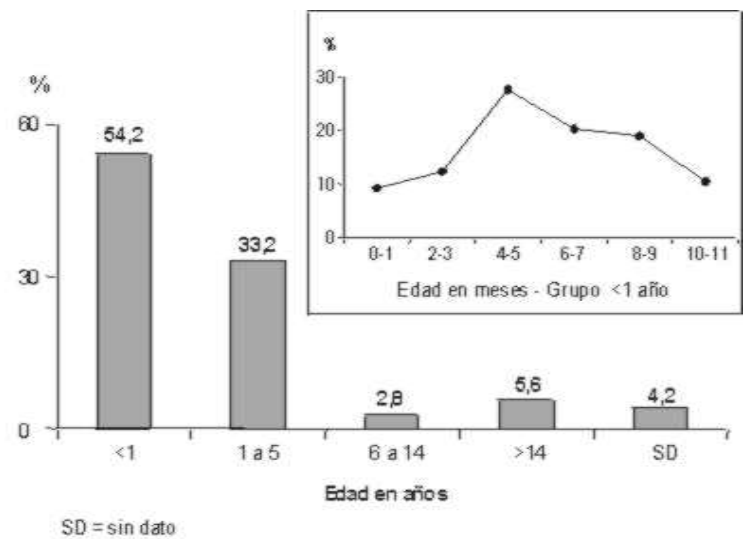

Figura 1. Distribución de los aislamientos invasores de Haemophilus influenzae por grupos de edad y distribución en el grupo de menores de 1 año.

de edad de mayores o iguales a 15 años, tanto el serotipo b como el NC estaban asociados con neumonía, $85,7 \%$ y $90,9 \%$, respectivamente (cuadro 2).

Durante los nueve años de vigilancia, se determinó una disminución en la frecuencia relativa del serotipo b; en 1996 representó el 98,3\% de los aislamientos y en 2001 , el $51,5 \%(p<0,001)$. En cuanto a $H$. influenzae NC, se incrementó de $1,7 \%$ en 1996 a $39,4 \%$ en 2001 ( $p<0,001)$; este último cambio estuvo asociado especialmente con el grupo de niños menores de 1 año, cuya frecuencia promedio en los primeros años de vigilancia, antes del inicio de la vacunación (1994-

Cuadro 1. Distribución de los aislamientos invasores de $H$. influenzae por procedencia y año de vigilancia.

\begin{tabular}{|c|c|c|c|c|c|c|c|c|c|c|c|}
\hline \multirow[b]{2}{*}{ Departamentos } & \multicolumn{11}{|c|}{ Años de vigilancia } \\
\hline & 1994 & 1995 & 1996 & $\begin{array}{c}1997 \\
\text { n }\end{array}$ & 1998 & 1999 & 2000 & 2001 & 2002 & & $\begin{array}{l}\text { tal } \\
(\%)\end{array}$ \\
\hline Bogotá & 32 & 33 & 42 & 56 & 61 & 30 & 13 & 8 & 7 & 282 & $(41,5)$ \\
\hline Antioquia & 4 & 18 & 26 & 35 & 9 & 9 & 20 & 16 & 20 & 154 & $(22,6)$ \\
\hline Valle & 0 & 20 & 25 & 9 & 2 & 1 & 0 & 0 & 3 & 60 & $(8,8)$ \\
\hline Santander & 0 & 0 & 1 & 14 & 23 & 8 & 5 & 3 & 2 & 56 & $(8,2)$ \\
\hline Risaralda & 0 & 4 & 12 & 12 & 12 & 5 & 6 & 2 & 1 & 54 & $(7,9)$ \\
\hline Norte de Santander & 2 & 4 & 11 & 7 & 0 & 0 & 0 & 0 & 1 & 25 & $(3,7)$ \\
\hline Caldas & 2 & 1 & 1 & 3 & 3 & 0 & 0 & 1 & 3 & 14 & $(2,1)$ \\
\hline Otros* & 0 & 2 & 2 & 5 & 9 & 4 & 4 & 3 & 6 & 35 & $(5,1)$ \\
\hline Total & 40 & 82 & 120 & 141 & 119 & 57 & 48 & 33 & 43 & 683 & \\
\hline
\end{tabular}

* Tolima (11), Amazonas (8), Nariño (5), Cundinamarca (3), Arauca (2), Atlántico (2), Caquetá (1), Magdalena (1), Sucre(1) y Vaupés (1).

$0=$ no se recibieron aislamientos. 
Cuadro 2. Distribución de los serotipos de los aislamientos invasores de H. influenzae con respecto al diagnóstico y grupos de edad.

\begin{tabular}{|c|c|c|c|c|c|c|c|c|c|c|c|}
\hline \multirow{4}{*}{$\begin{array}{l}\text { Edad en } \\
\text { años }\end{array}$} & \multirow{4}{*}{$\begin{array}{c}n \\
\text { total }\end{array}$} & \multicolumn{8}{|c|}{ Serotipos } & \multirow{3}{*}{\multicolumn{2}{|c|}{$\begin{array}{c}\text { Otros } \\
\text { serotipos }\end{array}$}} \\
\hline & & \multicolumn{4}{|c|}{$\mathbf{b}^{*}$} & \multicolumn{4}{|c|}{$N C^{\star *}$} & & \\
\hline & & \multicolumn{2}{|c|}{ meningitis } & \multicolumn{2}{|c|}{ neumonía } & \multicolumn{2}{|c|}{ meningitis } & \multicolumn{2}{|c|}{ neumonía } & & \\
\hline & & & $(\%)$ & & $(\%)$ & & (\%) & $\mathbf{n}$ & $(\%)$ & $\mathbf{n}$ & $\%$ \\
\hline$<1$ & 370 & 285 & $(77,0)$ & 48 & $(13,0)$ & 5 & $(1,4)$ & 21 & $(5,7)$ & 8 & $(2,2)$ \\
\hline $1-5$ & 227 & 147 & $(64,8)$ & 45 & $(19,8)$ & 9 & $(4,0)$ & 18 & $(7,9)$ & 3 & $(1,3)$ \\
\hline $6-14$ & 19 & 13 & $(68,4)$ & 2 & $(10,5)$ & 4 & $(21,1)$ & 0 & $(0,0)$ & 0 & $(0,0)$ \\
\hline$\geq 15$ & 38 & 2 & $(5,3)$ & 12 & $(31,6)$ & 2 & $(5,3)$ & 20 & $(52,5)$ & 2 & $(5,3)$ \\
\hline Sin dato & 29 & 18 & $(62,1)$ & 7 & $(24,1)$ & 2 & $(6,9)$ & 1 & $(3,4)$ & 1 & $(3,4)$ \\
\hline Total & 683 & 465 & $(68,1)$ & 114 & $(16,7)$ & 22 & $(3,2)$ & 60 & $(8,8)$ & 14 & $(2,0)$ \\
\hline
\end{tabular}

* De los aislamientos serotipo b, 2 eran de pacientes con sepsis ( 1 de $<1$ año y 1 de 1-5 años)

** De los aislamientos no capsulares 6 eran de pacientes con sepsis $(2$ de $<1$ año y 4 de $1-5$ años)

1998), era de $2,1 \%$ y pasó a $25,3 \%$ (1999 a 2002) $(p<0,001)$. El mismo fenómeno se presentó en el grupo de 1 a 5 años, que pasó de 13,6\% (1994$1998)$ a $33,8 \%(1999-2002)(p<0,001)$. En los dos grupos de edad, la mayoría de los aislamientos de $H$. influenzae NC eran de pacientes con neumonía. En el resto de los grupos no se observaron cambios significativos.

Susceptibilidad antimicrobiana. La vigilancia de los patrones de susceptibilidad antimicrobiana de los 683 aislamientos, durante el mismo período, mostró que $82(12 \%)$ fueron productores de $\beta$ lactamasa, $95(13,9 \%)$ presentaron resistencia a ampicilina, $87(12,7 \%)$ a SXT y $36(5,2 \%)$ a cloranfenicol. El estudio de la resistencia a cefalosporinas se realizó en 586 aislamientos, de los cuales $6(1 \%)$ fueron resistentes a cefuroxima. Todos los aislamientos fueron sensibles a ceftriaxona.

Al analizar la evolución de la resistencia en los 683 aislamientos, se encontró que en 1994, 10\% eran productores de $\beta$ lactamasa y en 2002, 14\% $(p=0,4)$. La resistencia a ampicilina pasó de $12,5 \%$ (7,5\% intermedia y $5 \%$ alta) en 1994 a $17,5 \%$ en 1999 y a $11,6 \%$ (2,3\% intermedia y $9,3 \%$ alta) en $2002(p=0,4)$. Para cloranfenicol, en 1994 la resistencia fue de $15 \%$ y en los últimos tres años (2000-2002), todos los aislamientos han sido sensibles. Para cefuroxima, se observó en los primeros años de la vigilancia una resistencia de $6,3 \%$, pero desde el 2000 hasta la fecha todos los aislamientos han sido sensibles.
En cuanto al SXT, la resistencia se incrementó de $5 \%$ en 1994 , a $13 \%$ en $2002(p=0,1)$, pero con grandes variaciones durante el período de vigilancia, alcanzando la mayor frecuencia relativa de resistencia en 1995 con $27,2 \%$ y la más baja en 2000 con $4,3 \%$.

Impacto de la vacuna. Al realizar el análisis se encontró que antes de 1999 se esperaba un promedio anual de 43 aislamientos en el grupo de los menores de 1 año y se recibieron 15 en 1999 , 17 en 2000, 8 en 2001 y 10 en 2002. En el grupo de 1 a 5 años, se esperaba recibir 23 aislamientos y se recibieron $12,9,5$ y 6 en cada uno de los cuatro años, respectivamente, lo que indica una disminución significativa en los dos grupos de edad $(p<0,001)$ (figura 2$)$.

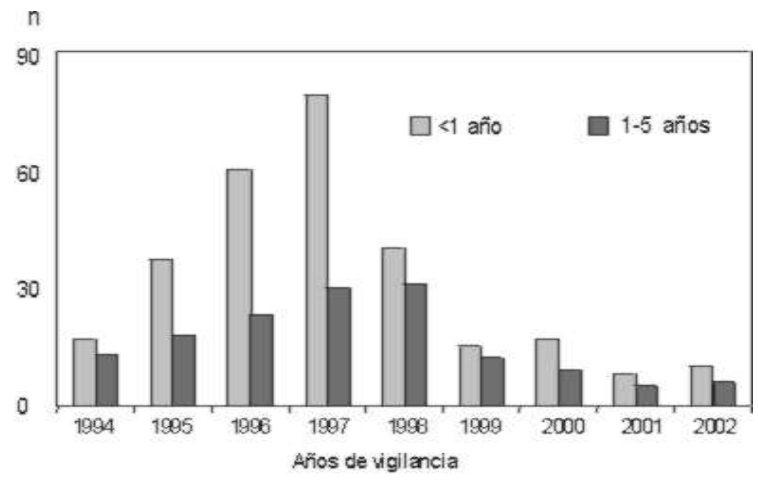

Figura 2. Número de casos de meningitis causada por Haemophilus influenzae, serotipo b, confirmados por el laboratorio en menores de 1 año y de 1 a 5 años (1994 al 2002). 


\section{Discusión}

Este sistema de vigilancia, realizado a través de los datos suministrados por los laboratorios desde 1994, ha permitido recopilar información específica sobre la evolución de los serotipos y los patrones de susceptibilidad antimicrobiana y determinar la disminución de los casos después de la vacunación, cumpliendo con los objetivos principales de este tipo de vigilancia (19).

Sin embargo, en nuestro medio los sistemas de vigilancia por el laboratorio tienen la desventaja de las coberturas. No obstante haberse recibido aislamientos de 17 departamentos, $94,8 \%$ de ellos provienen de 6 departamentos y el Distrito Capital, con la ventaja de que éstos son los departamentos más densamente poblados, lo cual equivale al $36 \%$ de la población total. Adicionalmente, se debe tener en cuenta que el desarrollo tecnológico de los LSP del país es diferente; existen LSP que no tienen un área de microbiología y, por tanto, les es muy difícil participar de la vigilancia.

En esta vigilancia, $H$. influenzae estuvo más asociado como agente de la meningitis, lo que se debió, posiblemente, a que el diagnóstico por el laboratorio de la meningitis es un procedimiento más sensible y menos costoso que permite una fácil recuperación del patógeno, comparado con el diagnóstico de la neumonía. Esta vigilancia mostró que $H$. influenzae afecta principalmente a la población pediátrica menor de 1 año y especialmente a los de 0 a 6 meses (33\%), lo cual indica la importancia de la vacunación en este grupo de edad. Estos datos son similares a los encontrados en un informe inicial del programa de vigilancia de meningitis bacteriana aguda (MBA), de 1994 a 1997, en el que $H$. influenzae, serotipo $b$, fue el patógeno aislado con mayor frecuencia en la población menor de 5 años (8).

Otros estudios sobre la etiología de las MBA, realizados en el Hospital Infantil de Medellín entre 1984 y 1986 y en el Laboratorio de Salud Pública (LSP) de Antioquia de 1990 a 1997, informaron que en el grupo de menores de un año, $48,9 \%$ de las meningitis eran causadas por $\mathrm{H}$. influenzae serotipo b $(20,21)$. Estos datos son semejantes a los informados por otros autores colombianos, como por los análisis realizados en otros países $(6,22)$.

De acuerdo con lo esperado, el serotipo predominante fue el $b$, implicado más frecuentemente en casos de meningitis, antes y después de la vacunación (1994-1998). Le siguió $H$. influenzae NC, asociado principalmente con neumonía. Es importante anotar el incremento significativo de los aislamientos $H$. influenzae NC en los menores de 5 años; Black y colaboradores, en un estudio de población realizado en California, observaron un incremento, aunque no significativo, de los $H$. influenzae NC causantes de enfermedad invasora (23).

En el informe realizado de la vigilancia entre 1994 y 1997 se observó que de 264 aislamientos estudiados, $13 \%$ eran resistentes a ampicilina, $7,6 \%$ a cloranfenicol, $27,3 \%$ a SXT, $1 \%$ a cefuroxima y que todos los aislamientos fueron sensibles a ceftriaxona (9). De igual manera, en la vigilancia realizada en el LSP de Antioquia, en los 147 aislamientos estudiados, se observó resistencia a ampicilina en 5,58\%, a SXT en $9,1 \%$ y a cloranfenicol en $3,4 \%(20,21)$. Sin embargo, el análisis de la vigilancia de la susceptibilidad antimicrobiana en este estudio demostró que los aislamientos colombianos son sensibles a los antibióticos de primera elección (1), con un incrementó de resistencia únicamente a SXT; para los otros antibióticos, la tendencia fue a la disminución; así, no se han presentado aislamientos resistentes durante los últimos tres años al cloranfenicol y a las cefalosporinas, por ejemplo. Estos cambios se deben posiblemente a la disminución del número de casos y a la disminución del empleo de estos antibióticos para el tratamiento (24).

Al realizar un nuevo análisis del impacto de la vacuna encontramos que el número de casos de meningitis por $H$. influenzae, serotipo $b$, ha disminuido significativamente desde la introducción de la vacuna, observación que ya se había informado en Colombia (15). Sin embargo, es importante anotar que la vacuna no ha tenido el impacto documentado en otros países $(12,13)$. Esto se debe posiblemente a que la cobertura de vacunación nacional hasta el 2001 no alcanzó el 
$60 \%$, con rangos en los departamentos de $17,7 \%$ a $92 \%$ (1).

De igual manera, durante 2002, fueron informados al SIVIGILA (Datos Minsalud), 110 casos de meningitis causadas por $\mathrm{H}$. influenzae. Si tenemos en cuenta que de 1994 a 2002 la vigilancia a través del laboratorio concluyó que $90,5 \%$ de los casos de meningitis por $H$. influenzae se dieron en menores de 5 años, se puede inferir que 99 de los 110 casos informados en 2002 fueron en este grupo edad, lo que indica nuevamente el problema de la baja cobertura de vacunación contra $H$. influenzae, serotipo b, en el país (1).

Finalmente, se puede observar que el sistema de vigilancia de la enfermedad invasora causada por $H$. influenzae a través del laboratorio ha permitido evaluar los cambios epidemiológicos antes y después del inicio de la vacunación y ha identificado la población de mayor riesgo, la evolución de los serotipos y los patrones de susceptibilidad antimicrobiana. Estos datos indican que, a pesar de las debilidades de los sistemas de vigilancia, sí proporcionan datos valiosos que pueden ser extrapolados a la población general y tenidos en cuenta dentro de la vigilancia en salud pública del país.

Por estas razones y, a pesar de la disminución en el número de casos, es muy importante seguir con mayor esfuerzo la vigilancia de $H$. influenzae, tanto para conocer los casos nuevos de enfermedad invasora, la aparición de serotipos diferentes del b y la resistencia antimicrobiana, así como para conocer el esquema de vacunación de cada uno de los casos y poder así reevaluar la cobertura de la vacunación y determinar sus fallas. Por esta razón, es necesario que todos los casos de enfermedad invasora por $H$. influenzae sean confirmados por el laboratorio de referencia y que incluyan los datos de vacunación para poder determinar si es o no falla en ésta. Esto sería de gran utilidad para la vigilancia de este patógeno.

\section{Referencias}

1. Gutiérrez C. Infecciones por Haemophilus influenzae en la población pediátrica. Biomédica 2001;21:369-88.

2. Shapiro ED, Ward JI. The epidemiology and prevention of disease caused by Haemophilus influenzae type b. Rev Epidemiol 1991;13:113-39.
3. Steinhoff M. Haemophilus influenzae tipo $\mathrm{b}$ infections are preventable everywhere. Lancet 1997;349:1186-7.

4. Murphy TV, White KE, Pastor P, Gabriel L, Medley F, Granoff DM, et al. Declining incidence of Haemophilus influenzae type $b$ disease since introduction of vaccination. JAMA 1993;13:246-8.

5. Peltola H. Worldwide Haemophilus influenzae type $b$ disease at the beginning of the 21st century: global analysis of the disease burden 25 years after the use of the polysaccharide vaccine and a decade after the advent of conjugates. Clin Microbiol Rev 2000;13:30217.

6. Ferrecio C, Ortiz E, Astroza L, Rivera C, Clemens J, Mirón L. A population-based retrospective assessment of the disease burden resulting from invasive Haemophilus influenzae in infants and young children in Santiago, Chile. Pediatric Infect Dis J 1990;9:488-93.

7. Doren GV, Jergensen JH, Thornsberry C. National collaborative study of prevalence of antimicrobial resistance among clinical isolates of Haemophilus influenzae. Antimicrob Agents Chemoter 1998;32:10815.

8. Agudelo $\mathrm{Cl}$, Muñoz N, Sanabria OM, Galindo B, García MJ y colaboradores. Etiología de la meningitis bacteriana aguda: programa de red de laboratorios, 1994-1997. Resúmenes, Congreso Internacional de Investigación y Salud, INS 80 años. Biomédica 1997; 17(Supl.1):109.

9. Muñoz N, Linares M, Agudelo Cl. Susceptibilidad antimicrobiana de Haemophilus influenzae como agente de la meningitis bacteriana aguda y neumonía. Resúmenes, Congreso Internacional de Investigación y Salud, INS 80 años. Biomédica 1997;17(Supl.1):109.

10. Ruocco G, Curto S, Savio M, Laurani H, Froncht R. Vacunación contra Haemophilus influenzae tipo b en el Uruguay: experiencia e impacto. Rev Panam Salud Pública 1999;5:197-206.

11. Lagos R, Horwitz I, Toro J, San Martín O, Abrego P, Bustamante $\mathbf{C}$, et al. Large scales post licensure, selective vaccination of Chilean infants with PRP-T conjugate vaccine: practicality and effectiveness in preventing invasive Haemophilus influenzae type b infections. Pediatr Infect Dis J 1996;15:216-22.

12. Adams WG, Deaver KA, Cochi SL, Plikaytis BD, Zella ER, Broome CV, et al. Decline of childhood Haemophilus influenzae type b (Hib) disease in the Hib vaccine era. JAMA 1993;269:221-6.

13. Agudelo C, Muñoz N, De la Hoz F. Evaluación rápida del impacto de la vacuna contra Haemophilus influenzae serotipo b en Colombia. Rev Panam Salud Pública 2000;8:181-4.

14. Higuera AB, De la Hoz F, Pastor D, Guisays G, Luna M, Gómez A, et al. Efectividad de la vacuna para Haemophilus influenzae, tipo b, (Hib) en neumonías 
bacterianas en Colombia. Inf Quinc Epidemiol Nac 2001; 6:238-9.

15. Koneman EW, Allen SD, Janda WM, Schreckenberger PC, Winn WC, Jr, editores. Haemophilus. Color atlas and textbook of diagnostic Microbiology. Fifth edition. Philadelphia, PA: Lippincot, Williams and Wilkins; 1997. p.363-93.

16. Campos MJ. Haemophilus. En: Murray PR, Baron EJ, Pfaller MA, Tenover FC, Yolken RH, editors. Manual of clinical microbiology. Seventh edition. Washington, D.C.: ASM Press; 1999. p.604-24.

17. National Committee for Clinical Laboratory Standards. Performance standards for antimicrobial testing; eleventh informational supplement; NCCLS document M100-S11. Wayne, Pennsylvania: NCCLS; 2001.

18. National Committee for Clinical Laboratory Standards. Methods for dilution antimicrobial susceptibility tests for bacteria that grow aerobically; approved standard. Fifth edition. M7-A5. Wayne, Pennsylvania: NCCLS; 2000.

19. Anchuela OT, Heraas CA, Pachón I, Martínez JF. Vigilancia de la salud pública. En: Martínez F, Antó JM, Castellanos PL, Gili M, Morset P, Navarro V, editores.
Salud pública. Madrid, España: McGraw-Hill Interamericana; 1997.

20. Otero R, Lux AM, Mejia J, Agudelo N, Zapata CJ, Hoyos D. Meningitis bacteriana aguda en niños. Estudio clínico y bacteriológico en el Hospital Infantil de Medellín. IATREIA 1998;2:69-75.

21. Jaramillo E, Estrada S. Resultados de la vigilancia de laboratorio en meningitis bacteriana, ocho años de seguimiento. Acta Med Colomb 1998;23:334-8.

22. Morales M, Dumar J, Eslava E, Estrada M, Zapata C, Agudelo $\mathrm{N}$, et al. Aspectos actuales de la meningitis bacteriana. Acta Neurol Colomb 2000;16:211-5.

23. Black SB, Shinefield RH, the Kaiser Permanent Pediatric Vaccine Study Group. Immunization with oligosaccharide conjugate Haemophilus influenzae type b (HbOC) vaccine on a large health maintenance organization population: extended follow-up and impact on conjugate Haemophilus influenzae disease epidemiology. Pediatr Infect Dis J 1992;11:610-3.

24. Zanella C, Casagrande S, Bokermann S, Almeida S, Brandileone MC. Characterization of Haemophilus influenzae isolated from invasive disease in Brazil from 1990 to 1999. Microb Drug Resist 2002;8:67-72. 